\title{
Perfil de produção acadêmica dos programas brasileiros de pós- graduação em Ciência da Informação 2008-2012
}

Marynice Medeiros Matos Autran

Professora do Departamento de Ciência da Informação -Universidade Federal da Paraíba Doutora em Informação e Comunicação em Plataformas Digitais - Universidade do Porto/Universidade de Aveiro - Portugal

Maria Manuel Borges

Professora do Departamento de Filosofia, Comunicação e Informação da Faculdade de Letras da Universidade de Coimbra, Portugal PhD em Ciência da Informação

Jesús Pascual Mena-Chalco

Professor do Departamento de Matemática e Estatística da Universidade Federal do ABC.Doutor em Ciência da Computação

Victor Guimarães Pinheiro

Professor do Instituto Paraibano de Educação (UNIPÊ) e da Faculdade de Ensino Superior do Nordeste (FAESNE). Especialista em Segurança da Informação

http://dx.doi.org/10.1590/1981-5344/2434

As práticas de comunicação da ciência dependem de culturas epistêmicas diferenciadas. A literatura relata inúmeras pesquisas, sob os mais diferenciados enfoques, relacionados aos programas brasileiros de pós-graduação em ciência da informação (PPGCIs). Nesta pesquisa esses programas foram caracterizados considerando o capital intelectual acumulado. Entretanto, o que se conhece sobre o perfil próprio de atuação para a produção de ciência nesses programas? Esta investigação visa: analisar a produção acadêmica - comunicações apresentadas em eventos, artigos de periódicos, capítulos de livros, livros organizados, editados, publicados $e$ resumos expandidos - no quinquênio 2008 a 2012. A metodologia incluiu a extração automática do Currículo 
Lattes dos docentes, efetivos e colaboradores dos PPGCIs, em atividade até 31 de dezembro de 2012. Para a extração foi utilizada a ferramenta scriptlattes. Dentre os resultados obtidos, foi evidenciado que a produção acadêmica se concentra, majoritariamente, em comunicações apresentadas em eventos $(36,8 \%)$ e artigos de periódicos (34,3\%). Adicionalmente, foi identificada a existência de três tipos de perfil/atuação dos PPGCIs para o quinquênio: (a) perfil estável de produção, (b) perfil com leve decremento de produção, e (c) perfil com leve incremento de produção.

Palavras-chave: Produção científica. Programas de pósgraduação em Ciência da Informação. ScriptLattes.

\section{Scholarly production of the Brazilian Information Science graduate programs in the period 2008-2012}

Scholarly communication practices depend on different epistemic cultures. The literature reports numerous studies, under the most different approaches related to Brazilian graduate programs in information science (PPGCIs). However, what is known about the profile of expertise for the production of science in these programs? In this research, the PPGCIs were characterized considering the intellectual capital accumulated. From this point of view, this research aims to: analyze the academic production - communications presented on events, journal articles, book chapters, books organized, edited, published and expanded abstracts in the period 2008 to 2012. The methodology included the automatic extraction from curriculum Lattes of permanent and temporary teachers, in activity until December 31, 2012. For extracting scriptlattes tool was used. The results indicate that the academic production focuses, largely in communications presented at events (36.8\%) and journal articles (34.3\%). Additionally, it was evidenced the existence of three types of profile/performance for the period: profile production stable, profile with a slight decrement of production and profile with a slight increase of production.

Keywords: Scientific production. Brazilian graduate programs in information Science. ScriptLattes. 


\section{Introdução}

A constituição da comunidade científica engloba dois fenômenos distintos: o social e o cognitivo, fatores estes que resultam na complexidade de sua concepção e conceituação.

Comunidades são organizações sociais que compreendem grupos, relações interpessoais, partilha de sentimentos, ideias, emoções, interesses, informações e comunicação. Sendo a comunicação um processo social básico, que norteia e regula as relações nesse corpo social, que é a comunidade, faz-se necessário que as "[...] pessoas sejam capazes de falar a sua linguagem." (ZIMAN, 1979, p. 58) advindo daí o aspecto social. O aspecto cognitivo, por sua vez, diz respeito à construção da ciência propriamente dita, ao desenvolvimento científico. Destarte, compreende-se o papel do cientista: "Cabe ao cientista tomar decisões, investigar, pois somente ele possui mente e consegue ter representações mentais da natureza." (SZCZEPANIK, 2013, p. 9).

Os estudos sobre comunidade científica têm sido objeto de atenção e abordagem de vários autores, como Polaniy (1946; 1968), Kuhn (1978), Le Coadic (2004), Meadows (1999), Schwartzman (2001), entre outros. O trabalho seminal de Ludwik Fleck, ${ }^{1}$ publicado em 1935, tráz à tona o conceito de "pensamento coletivo" também denominado "trabalho comunitário", que muito se assemelha aos conceitos atuais de comunidade científica. Isto sugere que as ideias de Fleck antecedem às de Polanyi, a quem se atribui cunhar o termo comunidade científica, imaginando-a como "uma grande e complexa república" à qual denominou de "República da Ciência", conceituando-a como uma sociedade de exploradores que lutam em prol de um futuro desconhecido, que acreditam ser acessível e digno de ser atingido. O cientista-explorador se esforça por aproximar-se de uma realidade oculta, para a sua satisfação intelectual. À medida que ele se satisfaz, ilumina todos os homens e ajuda assim a sociedade a cumprir suas obrigações, no sentido do autoaperfeiçoamento intelectual (POLANIY, 1968)

Na perspectiva de Schwartzman (2001) uma comunidade científica pode ser entendida nos seguintes termos:

Um grupo de indivíduos que compartilham valores e atitudes científicas, e que se inter-relacionam por meio das instituições científicas a que pertencem. Diz-se que uma comunidade científica é formada por indivíduos que têm em comum habilitações, conhecimentos e premissas tácitas sobre algum campo específico do saber. (SCHWARTZMAN, 2001, p. 23).

\footnotetext{
Médico e biólogo polonês (1896-1961). Publicou originalmente em alemão o livro intitulado Entstehung undEntwicklung einer wissenschaftlichen Tatsache: Einführung in die Lehre vom Denkstill und Denkkollektiv, traduzido para o inglês sob o título Genesis and Development of a Scientific Fact.
} 
Kuhn (1978) se reporta, explicitamente, a Fleck como antecessor de sua concepção de comunidade científica e reconhece a influência que ele exerceu sobre suas ideias. De acordo com seu ponto de vista, a comunidade científica tem como característica a reunião de um grupo com formação em um domínio científico comum, com o objetivo de construção coletiva da ciência, um locus apropriado para atribuir-lhe legitimidade e convergência de opinião acerca dos mesmos paradigmas. Nessa perspectiva, afirma: "Um paradigma é aquilo que os membros de uma comunidade partilham e, inversamente, uma comunidade científica consiste em homens que partilham um paradigma." (KUHN, 1978, p. 219).

Esta seria a comunidade científica ideal, imaginária, almejada por todos os que dela fazem parte. Tratando-se, porém, de uma estrutura social constituída de indivíduos com traços de personalidade individuais e imbuídos de sentimentos inerentes ao homo academicus, ${ }^{2}$ como emoções, afetos, anseios, atitudes, motivações, conflitos, disputas, expectativas, reconhecimento etc., fatores que, indiscutivelmente, motivam alterações comportamentais.

O comportamento dos investigadores se tornou objeto de estudo da sociologia desde o início do século XX; é, porém, a partir das investigações de Robert Merton que esse novo ramo do conhecimento toma vulto e "[...] torna-se o conceito central da sociologia da ciência." (SILVA, 2002, p. 69).

Ao estudar a conduta dos cientistas Merton (1973) explora o que os motiva, recompensa, intimida e estabelece as regras de comportamento ou regras mertonianas que compõem o ethos científico que regula as comunidades científicas.

Entretanto, mesmo não havendo unanimidade e observância aos imperativos preconizados por Merton, eles têm como objetivo precípuo o cumprimento rigoroso do ethos científico e devem ser percebidos como um padrão comportamental.

\section{Tribos e territórios}

As práticas de produção e comunicação da ciência dependem da cultura epistêmica dos diferentes campos científicos (KNORR-CETINA, 1999; BECHER; TROWLER, 2001; CRONIN, 2003), não fugindo à regra dessa configuração a Ciência da Informação. Perceber como os cientistas trabalham, interagem com seus pares, o que e como produzem, nos diferentes domínios, tem despertado a atenção de vários estudiosos. Isto significa dizer que "[...] os cientistas são uma espécie muito observada, tanto longe quanto perto de seu habitat natural." (CRONIN, 2003, p. 1).

Para se compreender o comportamento da comunidade acadêmica ou "tribos e territórios" (BECHER; TROWLER, 2001), foram utilizadas como bases teóricas estudos que têm como pano de fundo a comunidade científica brasileira; contudo, trabalhos correlatos sobre a temática que

\footnotetext{
${ }^{2}$ Ver BOURDIEU, P. Homo Academicus. Cambridge: Polity Press, 1988.
} 
enfocam diferentes níveis e perspectivas sejam em âmbito local, regional, nacional, internacional e disciplinar, não poderiam ser desconsiderados, porquanto se trata de uma literatura que fundamenta a presente pesquisa.

Dentre os estudos brasileiros que analisam as comunidades científicas, destacam-se os de Pinheiro (2003), cuja investigação teve como foco os recursos eletrônicos utilizados por pesquisadores brasileiros na geração de novos conhecimentos. Na área específica de Biblioteconomia e Ciência da Informação, apontam-se, também, os trabalhos de Daura e Galvão (2011), que analisaram o perfil dos docentes atuantes nos cursos de graduação em Ciência da Informação (Biblioteconomia, Arquivologia e Museologia) ministrados em 27 universidades públicas brasileiras, com 0 objetivo de analisar 0 desdobramento da interdisciplinaridade na formação dos professores. Oliveira (2011) investigou a formação docente e caracterizou os conteúdos programáticos (temáticas, disciplinas e referências bibliográficas) dos cursos de graduação em Biblioteconomia existentes em universidades brasileiras que possuem programas de pós-graduação em Ciência da Informação. Alves (2011) pesquisou a comunidade docente dos cursos de Biblioteconomia localizados na região nordeste do Brasil, com o objetivo de perceber a influência do Acesso Aberto (AA) - open access.

No âmbito internacional, Bastos e Silva (2009) analisaram o uso das redes multimídia no processo de construção das rotinas cognitivas e sociais de investigadores da comunidade científica portuguesa e da comunidade científica do nordeste brasileiro. Bomfá e Silva (2009) identificaram os recursos utilizados pela comunidade científica portuguesa para a obtenção da visibilidade científica, considerando as bibliotecas digitais, as unidades de transferência de tecnologia, os arquivos bibliográficos online, as unidades de investigação e as associações que apoiam a divulgação da ciência. A dissertação de Costa (2009) analisou os padrões de comunicação da ciência utilizados pela comunidade científica da Universidade do Minho (Portugal). Além desses, as teses seguintes aprofundam essas questões e desvelam as práticas, as percepções e o comportamento infocomunicacional dessas comunidades acadêmicas. A investigação de Silva (2002) identificou as implicações provocadas pelas Tecnologias de Informação e Comunicação nas dinâmicas cognitivas da comunidade científica portuguesa. Também voltado para essa temática, Borges (2006), em sua tese, analisou as transformações e impactos nas novas formas de produção, acesso, uso e partilha do conhecimento pela comunidade científica da Universidade de Coimbra discutindo as questões ligadas ao AA à informação científica. Identificam-se, portanto, os vários níveis que podem assumir esses estudos, do nível nacional ao nível disciplinar, reforçando o que preceitua Silva (2002, p. 70), que afirma: "Quanto mais se especificar a abrangência da comunidade científica, mais a análise terá um carácter micro." Considera-se que esta pesquisa se enquadra no âmbito nacional-disciplinar, por contemplarem todos os PPGCIs brasileiros na área de CI. 


\section{Estrutura da oferta dos programas de pós-graduação em CI (PPGCIs)}

É no contexto da existência de cursos regulares de pós-graduação em Ciência da Informação e da literatura científica por eles produzidos que se insere esta pesquisa, a qual propõe como objetivo geral: analisar a produção e comunicação da ciência dos programas brasileiros de pósgraduação (PPGCIs) em Ciência da Informação no quinquênio 2008-2012. Para atingir esse objetivo definiu-se como objetivos específicos: identificar os programas com maior produção e produtividade face ao corpo docente; determinar a tipologia preferencial para comunicação da ciência e verificar a regularidade anual da produção acadêmica ao longo do período considerado.

Neste contexto, foram considerados todos os Programas de pósgraduação, tanto acadêmico como profissional (Quadro 1).

Quadro 1 - Programas de Pós-Graduação em CI

\begin{tabular}{|c|c|c|}
\hline PPGCI & PROGRAMA & INSTITUIÇÃO \\
\hline UEL* & $\begin{array}{l}\text { Programa de Pós-Graduação em Ciência } \\
\text { da Informação e Mestrado Profissional em } \\
\text { Gestão da Informação }\end{array}$ & $\begin{array}{l}\text { Universidade Estadual de } \\
\text { Londrina }\end{array}$ \\
\hline UFBA & $\begin{array}{l}\text { Programa de Pós-Graduação em Ciência } \\
\text { da Informação }\end{array}$ & Universidade Federal da Bahia \\
\hline UFF & $\begin{array}{l}\text { Programa de Pós-Graduação em Ciência } \\
\text { da Informação }\end{array}$ & Universidade Federal Fluminense \\
\hline UFMG & $\begin{array}{l}\text { Programa de Pós-Graduação em Ciência } \\
\text { da Informação }\end{array}$ & $\begin{array}{l}\text { Universidade Federal de Minas } \\
\text { Gerais }\end{array}$ \\
\hline UFPB & $\begin{array}{l}\text { Programa de Pós-Graduação em Ciência } \\
\text { da Informação }\end{array}$ & Universidade Federal da Paraíba \\
\hline UFPE & $\begin{array}{l}\text { Programa de Pós-Graduação em Ciência } \\
\text { da Informação }\end{array}$ & $\begin{array}{l}\text { Universidade Federal de } \\
\text { Pernambuco }\end{array}$ \\
\hline UFRJ/IBICT & $\begin{array}{l}\text { Programa de Pós-Graduação em Ciência } \\
\text { da Informação }\end{array}$ & $\begin{array}{l}\text { Universidade Federal do Rio de } \\
\text { Janeiro -Instituto Brasileiro de } \\
\text { Informação em Ciência e } \\
\text { Tecnologia }\end{array}$ \\
\hline UFSC** & $\begin{array}{l}\text { Programa de Pós-Graduação em Ciência } \\
\text { da Informação }\end{array}$ & $\begin{array}{l}\text { Universidade Federal de Santa } \\
\text { Catarina }\end{array}$ \\
\hline UNB & $\begin{array}{l}\text { Programa de Pós-Graduação em Ciência } \\
\text { da Informação }\end{array}$ & Universidade de Brasília \\
\hline UNESP & $\begin{array}{l}\text { Programa de Pós-Graduação em Ciência } \\
\text { da Informação }\end{array}$ & Universidade Estadual Paulista \\
\hline UNIRIO A & $\begin{array}{l}\text { Programa de Pós-Graduação em Gestão de } \\
\text { Documentos e Arquivos - Mestrado } \\
\text { Profissional em Gestão de Documentos e } \\
\text { Arquivos }\end{array}$ & $\begin{array}{l}\text { Universidade Federal do Estado } \\
\text { do Rio de Janeiro }\end{array}$ \\
\hline UNIRIO B & $\begin{array}{l}\text { Programa de Pós-Graduação em } \\
\text { Biblioteconomia - Mestrado Profissional em } \\
\text { Biblioteconomia }\end{array}$ & $\begin{array}{l}\text { Universidade Federal do Estado } \\
\text { do Rio de Janeiro }\end{array}$ \\
\hline USP & $\begin{array}{l}\text { Programa de Pós-Graduação em Ciência } \\
\text { da Informação }\end{array}$ & Universidade de São Paulo \\
\hline
\end{tabular}

Fonte: AUTRAN (2015).

*O Mestrado Profissional teve início em 2013.

** O Doutorado teve início em 2013. 
De acordo com a classificação das áreas do conhecimento da CAPES $^{3}$ a subárea Ciência da Informação encontra-se na grande área Ciências Sociais Aplicadas I. Em cada subárea estão relacionados os cursos de pós-graduação aprovados e reconhecidos. ${ }^{4}$ Dos 15 Programas existentes na área, oito oferecem os níveis de mestrado e doutorado e sete, apenas o mestrado. Desses, 11 são caracterizados como mestrados acadêmicos e quatro como mestrados profissionais em diferentes áreas, nomeadamente Biblioteconomia, Gestão de Documentos e Arquivos, Gestão da Informação e Gestão de Unidades de Informação, e oito oferecem o doutorado.

Nesse universo convergem sete programas para a região sudeste, $(46 \%)$, quatro para a sul, $(27 \%)$, três para a nordeste, $(20 \%)$ e um para a centro-oeste, $(6,6 \%)$. Efetivamente, a região sudeste é a mais aquinhoada concentrando quatro doutorados $(26,66 \%)$, cinco cursos de mestrado acadêmico $(33,33 \%)$ e dois de mestrado profissional $(13,33 \%)$.

Conhecer a produção e comunicação da ciência desses programas se torna relevante porque "[...] fornece um mapeamento das contribuições, necessidades e déficits nas diversas áreas do conhecimento como também possibilitam políticas de pós-graduação." (DOMINGOS, 1999, p. 47). Ademais, como defende Ziman (1979, p. 116), "[...] a literatura sobre um determinado assunto é tão importante quanto o trabalho de pesquisa a que ele dá origem." Nesse sentido, Mueller, Campelo e Dias (1996, p. 1) referem que "[...] a existência da literatura talvez seja o requisito mais importante para o desenvolvimento da ciência," e esse desenvolvimento "perpassa pela produção científica, que parece ser consolidada a partir de estudos e análises dos suportes documentais que veiculam as pesquisas em cada área." (DUARTE; SILVA; ZAGO, 2004, p. 174).

Para Mueller, Campello e Dias (1996, p. 1) a maturidade de uma área do saber assenta num tripé que inclui tanto a produção quanto as condições para a sua produção e difusão, através da formação e de sociedades científicas:

Três características são indícios da maturidade de uma área do saber e marcam o seu grau de institucionalização e desenvolvimento: a existência de literatura científica e profissional, a existência de uma associação ou sociedade científica e a existência de cursos regulares para a formação de novos profissionais e de pesquisadores.

\section{A descoberta de conhecimento em plataformas digitais}

\footnotetext{
${ }^{3}$ Disponível $\mathrm{rea}=60700009 \&$ descricaoArea $=\mathrm{CI} \%$ CANCIAS+SOCIAIS+APLICADAS+\&descricaoAreaConhecimento $=\mathrm{CI} \% \mathrm{C}$ ANCIA+DA+INFORMA\%C7\%C3O\&descricaoAreaAvaliacao=CI\%CANCIAS+SOCIAIS+APLICADAS+I $>$. Acesso em: 14 ago. 2012.

${ }^{4}$ Disponíve

$<$ http://conteudoweb.capes.gov.br/conteudoweb/ProjetoRelacaoCursosServlet?acao=pesquisarIes\&codigoA rea $=60700009 \&$ descricaoArea $=\mathrm{CI} \%$ CANCIAS+SOCIAIS+APLICADAS+\&descricaoAreaConhecimento $=\mathrm{CI} \% \mathrm{C}$ ANCIA+DA+INFORMA\%C7\%C3O\&descricaoAreaAvaliacao=CI\%CANCIAS+SOCIAIS+APLICADAS+I > . Acesso em: 14 ago. 2012.
} 
As novas Tecnologias de Informação e Comunicação (TIC) possibilitaram a emergência das plataformas de governo eletrônico (eGov), as quais têm como uma das finalidades apoiar a gestão pública na tomada de decisão, tornar transparente a gestão pública através da Lei de Acesso à Informação, formular planos e políticas governamentais e auxiliar na construção de indicadores para a ciência, tecnologia e inovação.

Apesar de essas plataformas disponibilizarem informações públicas, explícitas, o conhecimento implícito não é, ainda, devidamente extraído e explorado. Para tornar esse conhecimento explícito, técnicas têm sido desenvolvidas tais como Knowledge Discovery in Data Bases (KDD) e Data Mining. (FAYYAD; PIATETSKY-SHAPIRO; SMYTH, 1996).

Concentrada em um único Sistema de Informação, a Plataforma Lattes é uma iniciativa do Conselho Nacional de Pesquisa e Desenvolvimento Tecnológico (CNPq) e integra as bases de dados de Currículos, Grupos de Pesquisa e Instituições. Por serem públicos, os dados da Plataforma Lattes oferecem maior transparência e confiabilidade às

[...] atividades de fomento do CNPq e das agências que a utilizam, fortalecem $o$ intercâmbio entre pesquisadores e instituições e é fonte inesgotável de informações para estudos e pesquisas. Na medida em que suas informações são recorrentes e cumulativas, têm também o importante papel de preservar a memória da atividade de pesquisa no país. ${ }^{5}$

O Currículo Lattes (CL) tornou-se padrão nacional por ser um repositório que disponibiliza informação e conhecimento sobre ciência, tecnologia e inovação, além de registrar o histórico das atividades profissionais e acadêmicas, atuais e pregressas, dos pesquisadores cadastrados. Essas informações podem ser utilizadas como apoio à gestão, à formulação de políticas para C\&T, para avaliação da pesquisa acadêmica, além de ser um "[...] elemento indispensável e compulsório à análise de mérito e competência dos pleitos de financiamentos na área de ciência e tecnologia." (ALVES; YANASSE; SOMA, 2011a, p. 3) Além do português, o $\mathrm{CL}$ também possui versão em inglês e nos idiomas dos países que adotaram o modelo, nomeadamente Argentina, Chile, Colômbia, Cuba, Equador, México, Panamá, Paraguai, Peru, Portugal e Venezuela. (ALVES; YANASSE; SOMA, 2011b; ALVES; YANASSE; SOMA, 2011c).

Em artigo publicado na revista Nature, Julia Lane, diretora da Science of Science \& Innovation Policy da National Science Foundation dos Estados Unidos, cita o Currículo Lattes como uma das bases de dados de pesquisa mais completas e qualificadas (LANE, 2010).

A Engenharia do Conhecimento inclui a descoberta, modelagem e representação do conhecimento e apresenta uma série de ferramentas que podem revelar, por exemplo, dinâmicas de pesquisa e formação

\footnotetext{
${ }^{5}$ Disponível em: <http://lattes.cnpq.br/conteudo/aplataforma.htm>. Acesso em: 18 ago. 2013.
} 
profissional, redes de cooperação e socialização, tendências de criação e de inter-relações de áreas do conhecimento (PACHECO; KERN; STEIL, 2007).

O conhecimento implícito existente na Plataforma Lattes tem despertado interesse de pesquisadores nas mais diversas áreas do conhecimento. Baseadas nos dados do Currículo Lattes algumas pesquisas tiveram como alvo analisar o perfil dos pesquisadores que possuem bolsa de produtividade em pesquisa do CNPq. Das áreas em evidência destacam-se os pesquisadores contemplados nas áreas de Saúde Coletiva (BARATA; GOLDBAUM, 2003; SANTOS et al., 2009), Medicina (MARTELLIJUNIOR et al., 2010), Odontologia (SCARPELLI et al., 2008), Nefrologia e Urologia (CAVALCANTE et al., 2008), Química (SANTOS; CÂNDIDO; KUPPENS, 2010). Outros estudos baseados nos dados do Currículo Lattes foram realizados por Pacheco, Forcellini e Kern (2007), os quais trabalharam a construção de mapas do conhecimento da área de Engenharia Mecânica e por Silva et al (2012b) cujo estudo comparou três programas de pós-graduação em CI localizados na região nordeste. Um dos problemas relatados na literatura referentes à coleta de informações na base de dados Lattes é a extração de dados. Cavalcante et al., (2008) revelam a dificuldade para extrair manualmente informações do Currículo Lattes. Referem os autores que a coleta dos dados demorou quase três anos para analisar 132 currículos. Dessa forma, o processo manual é moroso e passível de erros, uma vez que não se usa nenhum mecanismo que automatize a extração de dados com base em rotinas que utilizem práticas da Engenharia do Conhecimento.

Alves; Yanasse; Soma (2011c) desenvolveram a ferramenta "LattesMiner", uma interface de programação de aplicativos (API) orientada a objetos, para extrair dados de qualquer pesquisador ou grupos de pesquisa registrados na Plataforma Lattes. Outra utilidade dessa ferramenta é a alimentação do Sistema SUCUPIRA, também desenvolvido por Alves, Yanasse e Soma (2011a).

Digiampietri e Silva (2011) criaram uma estrutura para construção e visualização de redes sociais acadêmicas a partir de dados extraídos do CL e categorizaram essas redes através de métricas de produtividade.

A ferramenta scriptLattes é um software open source com Licença Pública Geral. Trata-se de um sistema que vem sendo largamente utilizado por universidades brasileiras, agências de fomento e de pesquisa de várias instituições nacionais. O software tem como finalidade extrair e compilar grande volume de dados sobre as atividades acadêmicas dos pesquisadores com registro no Currículo Lattes. Na concepção da ferramenta foram planejados módulos com finalidades distintas, nomeadamente: 1) seleção de dados; 2) extração de dados; 3) tratamento de redundâncias; 4) geração de redes de coautoria; 5) computação de AuthorRank. O módulo 2 extrai a produção acadêmica, técnica e artística, através de um parser computacional ${ }^{6}$, previamente

\footnotetext{
${ }^{6}$ Programa baseado em análise textual que permite identificar e extrair regiões ou trechos específicos de texto.
} 
estabelecido por tipologia e de acordo com o período de tempo estipulado pelo interessado (MENA-CHALCO; CESAR-JUNIOR, 2009).

Considerando que o $\mathrm{CL}$ constitui uma fonte rica para explorações bibliométricas e cientométricas de produção acadêmica e, ainda, tomando como base as experiências de Santana et al. (2011); Mena-Chalco e Cesar-Junior (2011b); Danuello e Oliveira (2012); Silva et al. (2012b); Mena-Chalco, Digiampietri e Oliveira (2012) (MENA-CHALCO; DIGIAMPIETRI; CESAR-JUNIOR, 2012), optou-se pela utilização dessa ferramenta para "[...] explorar, identificar ou validar padrões de atividades científicas, trazendo assim informação bibliométrica e/ou cientométrica sobre um grupo de interesse." (DANUELLO; OLIVEIRA, 2012, p. 70).

\section{Procedimentos metodológicos}

O locus de análise teve como referência a Plataforma Lattes, através da qual se levantou o Currículo Lattes individual do docente, efetivo e colaborador, associado a cada programa de pós-graduação em Ciência da Informação, em plena atividade acadêmica até 31 de dezembro de 2012. Para também incluir o docente colaborador partiu-se da premissa de que estes contribuem com aportes teóricos e metodológicos importantes para a área, estão inseridos em uma linha de pesquisa, orientam teses e dissertações e possuem produção científica relevante para a área. Nesse levantamento foram identificados 217 docentes, permanentes e colaboradores, os quais constituem o corpus de análise desta investigação. As etapas da pesquisa seguiram a seguinte metodologia: a) identificação dos programas de pós-graduação; b) identificação dos professores permanentes e colaboradores, associados a cada programa de pós-graduação; c) registro dos identificadores individuais dos currículos Lattes de cada professor; d) extração da produção acadêmica de cada programa (produções bibliográficas), o que foi feito através da ferramenta scriptlattes.

A tipologia documental incluiu: artigos de periódicos, comunicações apresentadas em eventos, capítulo de livros, livros editados/organizados/publicados e resumos expandidos.

Todos os PPGCIs, tanto acadêmico como profissional foram considerados. Nessa questão há que se esclarecer que, inicialmente, a estratégia era não considerar determinados programas acadêmicos, como os da UFRJ/IBICT e UFF, cujas razões explicaremos adiante e, também, os programas de mestrado profissional, nomeadamente os da UNIRIO A, UNIRIO B e UEL, por se tratarem de cursos mais recentes. Contudo, quando se executou o pré-teste da extração automática percebeu-se que a magnitude da produção científica destes programas poderia ser comparada aos demais, tomou-se a decisão de incluí-los.

Atentou-se para uma particularidade do mestrado profissional da UEL que, apesar de manter uma página própria, em separado do mestrado acadêmico possui, em sua grande maioria, o mesmo corpo docente. Dessa 
forma, decidiu-se considerá-los um único programa como medida preventiva para evitar a duplicidade na produção científica.

Quanto aos PPGCIs da UFF e da UFRJ/IBICT, Silva et al. (2012a, p.11) optaram por não incluí-los porque à época "[...] esses programas passaram por alterações em sua constituição que comprometeriam uma análise mais precisa das relações de colaboração com a comunidade."

Não foram incluídos o mestrado profissional da Universidade do Estado de Santa Catarina (UDESC) nem o doutorado da Universidade Federal de Santa Catarina, em virtude do início de ambos ter ocorrido em 2013. Apenas fez-se menção a esses cursos para atualização do número de programas existentes.

\section{Revelações geradas da coleta de dados}

Para analisar o comportamento da produção científica foi utilizada a metodologia proposta por Silva et al. (2012b) isto é, calculou-se a média (QtProd/Per.) e o desvio padrão.

A extração automática através do scriptlattes resultou em 4855 documentos. Contudo, a partir de um cotejo manual, foram constatadas algumas duplicações (Tabela 1), apesar de a ferramenta possuir o módulo de tratamento de redundância. Essas duplicações ocorreram devido às inconsistências no nome dos autores, nos títulos dos documentos, na inclusão da tipologia documental, por exemplo. Foi verificado, também, que na extração da produção anual de livros, vários documentos não apresentavam data de publicação. Para evitar distorções foram considerados para análise apenas os livros identificados com data.

Em todos os suportes ocorreram diferenças, computando-se para a pesquisa manual o total de 4626 documentos, ou seja, uma diferença de 229 documentos, o que representa 4,7\% da extração automática. Este é um percentual significativo e demonstra a robustez da ferramenta, uma vez que, em se tratando de uma porcentagem inferior a 5\%, é um resultado que permite legitimar a utilização do scriptLattes.

Tabela 1 - Extração automática versus cotejo manual

\begin{tabular}{lcc}
\hline \multicolumn{1}{c}{ Tipologia Documental } & scriptLattes & Cotejo manual \\
\hline \hline Comunicações em eventos & 1696 & 1699 \\
Capítulo de livros & 943 & 948 \\
Livros organizados, publicados ou editados & 444 & 196 \\
Resumos expandidos & 196 & 197 \\
Artigos de periódicos & 1576 & 1586 \\
Total & $\mathbf{4 8 5 5}$ & $\mathbf{4 6 2 6}$ \\
Diferença & & $\mathbf{2 2 9}$ \\
\hline
\end{tabular}

Fonte: AUTRAN (2015).

De acordo com esse resultado, a produção acadêmica total dos PPGCIs no período 2008 a 2012 atingiu o patamar de 4626 publicações. 


\section{Análise e discussão dos resultados}

Os dados da produção científica são reproduzidos no Gráfico 1, onde os eixos representam os PPGCIs e cada linha está associada a uma tipologia documental. Dessa forma, pode-se comparar o tipo de publicação que mais produz cada PPGCI. As comunicações apresentadas em eventos detêm a maior incidência (1699) ou $36,8 \%$ da tipologia produzida, por ordem de frequência, pelos PPGCIs da UNESP, UFMG e UFPB. Os artigos de periódicos constituem o segundo suporte com maior ocorrência (1586), o que equivale a $34,3 \%$, repetindo-se o maior número de artigos nos programas supracitados. Para a tipologia capítulo de livros (948), destacam-se os PPGCIs da UNESP e USP, enquanto que na produção de livros (196), a maior incidência ocorre no programa da UNB. Por outro lado, os resumos expandidos (197) foram produzidos em maior escala pelos PPGCIs da UNESP, UFMG e UEL perfazendo $4,2 \%$.

Dos cinco suportes que compõem a tipologia documental, o programa da UNESP apresenta maior produção em quatro, constituindo-se o mais produtivo de todos os PPGCIs.

Gráfico 1 - Produção acadêmica dos PPGCIs no período 2008-2012

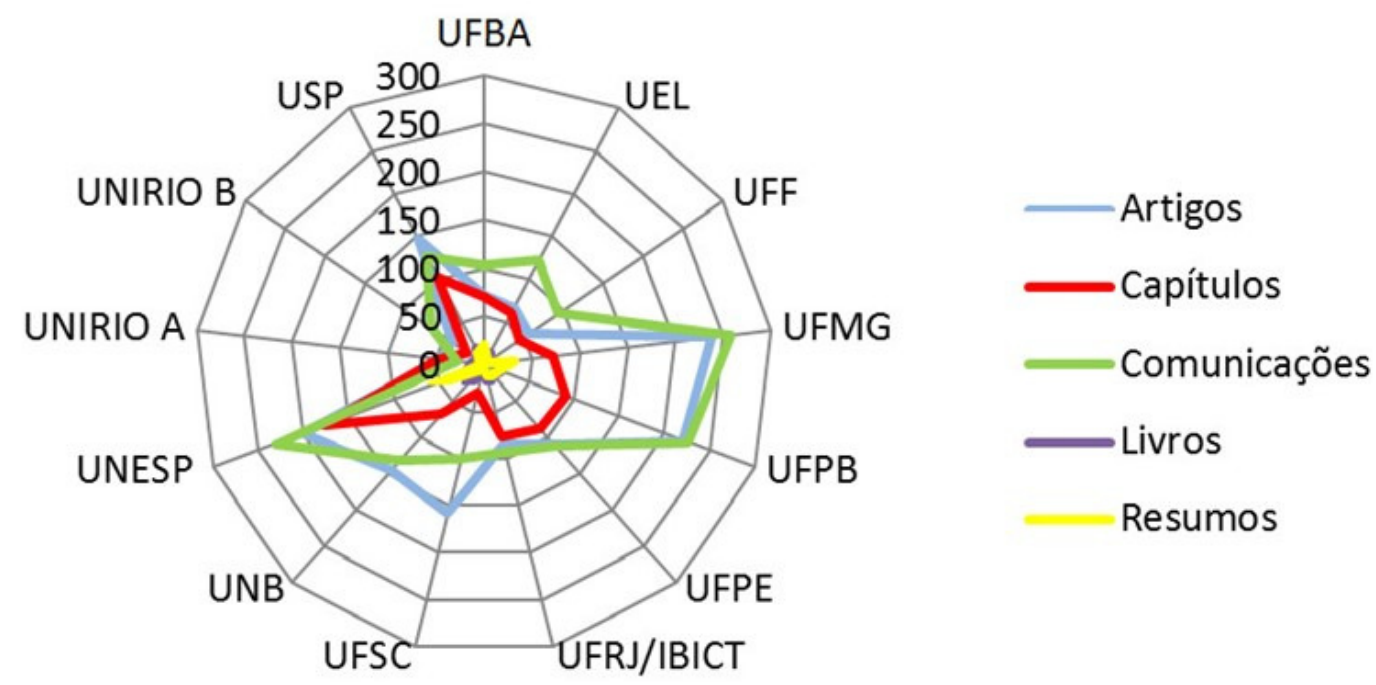

Fonte: AUTRAN (2015).

A demonstração da tipologia documental referente a cada programa tem como finalidade a comparação individual de cada componente da produção científica.

Apesar de se configurarem como um veículo de disseminação rápida para investigações em andamento ou recém-concluídas e servindo também para registrar previamente a autoria da publicação, os resumos expandidos não se revelaram um importante canal de comunicação da ciência. Essa afirmação se baseia na quantidade de resumos produzidos, com um total de 196 , ou $4,23 \%$ da produção total. Tradicionalmente, o livro se configura como o veículo de comunicação predominante na área 
das Ciências Humanas e Sociais (FUNDAÇÃO DE AMPARO À PESQUISA DO ESTADO DE SÃO PAULO, 2010; TARGINO; GARCIA, 2000). Contudo, afirmam Danuello; Oliveira (2012) que por serem os artigos de periódico considerados como indicador da qualidade da produção científica pelos órgãos de avaliação e de fomento, isso tem contribuído para o declínio da produção de livros.

Os números identificados nesta pesquisa permitem afirmar que a edição, organização e publicação de livros não é expressiva quando comparada às demais tipologias documentais. Neta tipologia o programa da UNB apresentou a maior produção/edição/organização de livros $(14,21 \%)$.

Os capítulos de livros revelam-se um importante canal de comunicação científica. A análise dessa tipologia demonstra que os PPGCIS produziram 948 capítulos, o que equivale a 20,5\% da produção total no quinquênio. Nessa tipologia destacam-se os PPGCIs da-UNESP, com 177 produções e da USP com 103 diferenciando-se, assim, dos demais programas.

Segundo Meadows (1999) as comunicações apresentadas em eventos são mais comuns na área das engenharias, onde os trabalhos apresentados são avaliados pelos pares e se equivalem aos artigos de periódicos. Em pesquisa realizada sobre os canais preferenciais para publicação Mueller (2005) conclui que "[...] para as Engenharias, os anais de congressos são canais prestigiosos e frequentes na comunicação da área," e que "[...] os pesquisadores da área das Engenharias confirmam a literatura, dando clara preferência aos congressos nacionais e estrangeiros." Com relação às comunicações em eventos na área de Ciência da Informação Noronha e Población (2002) afirmam que as comunicações em eventos concentram-se, principalmente, no Encontro Nacional de Pesquisa em Ciência da Informação (EnANCIB), Congresso Brasileiro de Biblioteconomia e Documentação (CBBD) e Seminário Nacional de Bibliotecas Universitárias (SNBU).

Foi constatado nesta investigação que os PPGCIs apresentaram 1699 comunicações em eventos, o que representa $36,72 \%$ da produção científica. A maior incidência ocorreu no PPGCI da UFMG perfazendo 258 comunicações. O mesmo comportamento verificou-se no PPGCI da UNESP que totalizou 231 publicações. Por sua vez, o PPGCI da UFPB apresentou crescimento considerável no biênio 2008-2010, iniciando com 23 e atingindo 60 comunicações em 2010, com a média de 45,2 comunicações/ano, porém com aparente oscilação entre 2011-2012. Estes programas apresentaram os maiores percentuais de produção de comunicações em eventos: PPGCI da UFMG $(15,18 \%)$, UNESP $(13,59 \%)$ e UFPB $(13,3 \%)$.

Legitimado pela comunidade científica como o veículo preferencial para a comunicação da ciência, "[...] os artigos de periódicos cumprem a função de comunicar resultados de pesquisas de forma rápida e sucinta." (DANUELLO; OLIVEIRA, 2012, p. 72). 
A produção de artigos no quinquênio atingiu o patamar de 1586, o que equivale a média de 317,2 artigos/ano, constituindo-se como a segunda maior em volume de produção. Nessa tipologia os PPGCIs com as maiores médias de produção/ano são: UFMG $(47,8)$, UFPB $(44,2)$ e UNESP (40) os quais publicaram, respectivamente, 238, 221 e 200 artigos.

Esse resultado permite evidenciar que o perfil de produção científica dos PPGCIs se caracteriza por publicar, no período observado, preferencialmente: comunicações apresentadas em eventos e artigos de periódicos. Isto permite também afirmar que, embora com uma pequena proporção, houve uma mudança no comportamento dos docentes em relação à escolha do canal preferencial para comunicação da ciência.

A Tabela 2 demonstra a produção anual, a média e o desvio padrão dos PPGCIs englobando toda a tipologia documental, onde se verifica que os Programas mais produtivos são UNESP, UFMG e UFPB.

Tabela 2 -Produção acadêmica dos PPGCIs por tipologia documental 2008-2012

\begin{tabular}{|c|c|c|c|c|c|c|c|c|c|c|c|c|c|c|}
\hline $\begin{array}{l}\text { Tipologia } \\
\text { documental }\end{array}$ & ANOS & UEL & UFBA & UFF & UFMG & UFPB & UFPE & $\begin{array}{l}\text { UFRJ } \\
\text { IBICT } \\
\end{array}$ & UFSC & UNB & UNESP & $\begin{array}{l}\text { UNIRIO } \\
\text { A }\end{array}$ & $\begin{array}{l}\text { UNIRIO } \\
\text { B }\end{array}$ & USP \\
\hline \multirow{8}{*}{$\begin{array}{l}\text { Resumos } \\
\text { expandidos }\end{array}$} & 2008 & 1 & 2 & 1 & 6 & 2 & 1 & 3 & -- & 3 & 12 & -- & 2 & 1 \\
\hline & 2009 & 6 & 1 & -- & 3 & 1 & 2 & 2 & -- & 1 & 2 & -- & -- & 2 \\
\hline & 2010 & 5 & 2 & 1 & 7 & 8 & 3 & -- & -- & 1 & 11 & -- & -- & -- \\
\hline & 2011 & 8 & -- & 2 & 7 & -- & 4 & 3 & 1 & 1 & 30 & 1 & 1 & 6 \\
\hline & 2012 & -- & 1 & 1 & 9 & 5 & 4 & 4 & 2 & 3 & 4 & -- & 2 & 5 \\
\hline & Total & 20 & 6 & 5 & 32 & 16 & 14 & 12 & 3 & 9 & 59 & 1 & 5 & 14 \\
\hline & Média & 4 & 1,2 & 1 & 6,4 & 3,2 & 2,8 & 2,4 & 0,6 & 1,8 & 11,8 & 0,2 & 1 & 2,8 \\
\hline & $\mathrm{DP}^{*}$ & 3,4 & 0,83 & 0,7 & 2,19 & 3,27 & 1,3 & 1,51 & 0,89 & 1,1 & 11,05 & 0,44 & 1 & 2,6 \\
\hline \multirow{8}{*}{$\begin{array}{l}\text { Livros } \\
\text { publicados, } \\
\text { organizados } \\
\text { ou editados }\end{array}$} & 2008 & 7 & 2 & -- & -- & 4 & 2 & 2 & -- & 5 & 3 & 2 & 2 & 5 \\
\hline & 2009 & -- & 6 & -- & 3 & 3 & 1 & 3 & 2 & 3 & 7 & 4 & 2 & 2 \\
\hline & 2010 & 4 & 2 & 2 & 3 & 4 & 4 & 5 & 6 & 8 & 2 & 2 & -- & 3 \\
\hline & 2011 & -- & 2 & -- & 6 & 3 & 7 & 2 & -- & 8 & 2 & 2 & 1 & 3 \\
\hline & 2012 & 3 & 2 & 1 & 5 & 5 & 1 & 7 & 1 & 4 & 5 & 4 & 2 & 6 \\
\hline & Total & 14 & 14 & 3 & 17 & 19 & 15 & 19 & 9 & 28 & 19 & 14 & 7 & 19 \\
\hline & Média & 2,8 & 2,8 & 0,6 & 3,4 & 3,8 & 3 & 3,8 & 1,8 & 5,6 & 3,8 & 2,8 & 1,4 & 3,8 \\
\hline & DP & 3 & 1,78 & 0,9 & 2,3 & 0,83 & 2,55 & 2,16 & 2,49 & 2,3 & 1,16 & 1,09 & 0,89 & 1,6 \\
\hline \multirow{8}{*}{$\begin{array}{l}\text { Capítulos de } \\
\text { livros }\end{array}$} & 2008 & 23 & 6 & 4 & 12 & 11 & 16 & 15 & 4 & 10 & 43 & 7 & 3 & 22 \\
\hline & 2009 & 5 & 17 & 5 & 11 & 14 & 16 & 10 & 6 & 6 & 26 & 7 & 6 & 20 \\
\hline & 2010 & 23 & 15 & 13 & 8 & 21 & 14 & 19 & 9 & 14 & 45 & 6 & 4 & 26 \\
\hline & 2011 & 5 & 15 & 6 & 18 & 23 & 20 & 11 & 9 & 24 & 20 & 10 & 4 & 20 \\
\hline & 2012 & 15 & 7 & 16 & 23 & 21 & 21 & 21 & 2 & 13 & 43 & 19 & 5 & 15 \\
\hline & Total & 71 & 60 & 44 & 72 & 90 & 87 & 76 & 30 & 67 & 177 & 49 & 22 & 103 \\
\hline & Média & 14 & 12 & 8,8 & 14,4 & 18 & 17,4 & 15,2 & 6 & 13,4 & 35,4 & 9,8 & 4,4 & 21 \\
\hline & DP & 9 & 5,09 & 5,4 & 6,02 & 5,19 & 2,96 & 4,81 & 3,08 & 6,69 & 11,54 & 5,35 & 1,14 & 4 \\
\hline \multirow{6}{*}{$\begin{array}{c}\text { Comunicações } \\
\text { em eventos }\end{array}$} & 2008 & 16 & 18 & 7 & 43 & 23 & 19 & 21 & 23 & 42 & 41 & 10 & 11 & 22 \\
\hline & 2009 & 18 & 23 & 18 & 50 & 41 & 19 & 27 & 17 & 32 & 48 & 6 & 12 & 28 \\
\hline & 2010 & 24 & 26 & 14 & 51 & 60 & 25 & 14 & 29 & 26 & 52 & 3 & 17 & 26 \\
\hline & 2011 & 32 & 32 & 23 & 51 & 55 & 34 & 16 & 17 & 21 & 51 & 6 & 12 & 31 \\
\hline & 2012 & 14 & 24 & 32 & 63 & 47 & 16 & 16 & 15 & 11 & 39 & 4 & 15 & 20 \\
\hline & Total & 104 & 123 & 94 & 258 & 226 & 113 & 94 & 101 & 132 & 231 & 29 & 67 & 127 \\
\hline
\end{tabular}




\begin{tabular}{c||lccccccccccccc} 
& Média & 21 & 24,6 & 19 & 51,6 & 45,2 & 22,6 & 18,8 & 20,2 & 26,4 & 46,2 & 5,8 & 13,4 & 25 \\
& DP & 7,3 & 5,07 & 9,4 & 7,19 & 14,4 & 7,16 & 5,26 & 5,76 & 11,6 & 5,89 & 2,68 & 2,5 & 4,5 \\
\hline \hline \multirow{5}{*}{ Artigos de } & 2008 & 12 & 17 & 11 & 36 & 34 & 19 & 12 & 27 & 32 & 27 & 9 & 6 & 25 \\
periódicos & 2009 & 15 & 13 & 9 & 47 & 27 & 12 & 26 & 35 & 35 & 31 & 12 & 10 & 28 \\
& 2010 & 13 & 10 & 13 & 53 & 43 & 21 & 12 & 33 & 21 & 37 & 8 & 8 & 29 \\
& 2011 & 20 & 13 & 16 & 53 & 50 & 30 & 15 & 38 & 35 & 60 & 7 & 7 & 41 \\
& 2012 & 12 & 14 & 7 & 49 & 67 & 27 & 19 & 26 & 22 & 45 & 10 & 10 & 24 \\
& Total & 72 & 67 & 56 & 238 & 221 & 109 & 84 & 159 & 145 & 200 & 47 & 41 & 147 \\
& Média & 14 & 13,4 & 11 & 47,8 & 44,2 & 21,8 & 16,8 & 31,8 & 29 & 40 & 9,4 & 8,2 & 29 \\
& DP & 3,4 & 2,5 & 3,8 & 7,19 & 15,5 & 7,04 & 5,89 & 5,16 & 47,8 & 13,07 & 1,67 & 1,78 & 6,8 \\
\hline \hline
\end{tabular}

Fonte: AUTRAN (2015).

*DP $=$ Desvio-padrão

A fim de identificar possíveis padrões na forma de atuação, foram subdivididos todos os PPGCIs em quatro grupos em função da ordem ou quantidade de publicações. O Gráfico 2a representa o desempenho dos programas mais produtivos (UFMG, UFPB e UNESP). Estes mantiveram um aparente crescimento linear na produção científica. Entretanto, o PPGCI da UNESP evidenciou decremento em 2012. Possivelmente isto tenha como causa a falta de atualização dos currículos, a periodicidade dos periódicos onde os artigos foram submetidos ou ainda o não cumprimento da periodicidade pelas revistas.

Com a finalidade de comparar os PPGCIs que apresentavam número de produções aproximadas, como por exemplo: com produção entre 330 e 410 (Gráfico 2b), foi analisada a linearidade ou oscilação ao longo dos anos. Nessa condição destacam-se os PPGCIs UFPE, UNB e USP, os quais mantiveram crescimento até 2011. Sem exceção, todos evidenciaram um aparente declínio em 2012. Para esses programas as médias de produção são: UFPE $(67,6)$, UNB $(76,2)$ e USP $(82)$.

Com escala de produção entre 200 e 400, encontram-se quatro programas, UEL, UFBA, UFRJ/IBICT e UFSC (Gráfico 2c), os quais apresentaram oscilações. Nestes, o único a apresentar crescimento em 2012 foi o PPGCI da UFRJ/IBICT. Os resultados demonstram que a média de produção dos PPGCIs UEL foi 56,2, UFBA, 54, UFRJ/IBICT, 57 e UFSC, 60,4 .

Evidenciando crescimento contínuo desde 2008, o programa da-UFF destaca-se neste grupo, do qual também fazem parte UNIRIO A e UNIRIO B (Gráfico 2d). Apesar de todos os programas apresentarem produção que oscila entre 100 e 200, o programa da UNIRIO A mantém linearidade desde o ano de 2010 e o da UNIRIO B, a partir de 2012. A produção média observada é de 40,6 (UFF), 23 (UNIRIO A) e 28,4 (UNIRIO B). 
Gráfico 2 - Evolução da produção acadêmica dos PPGCI no período 2008-2012

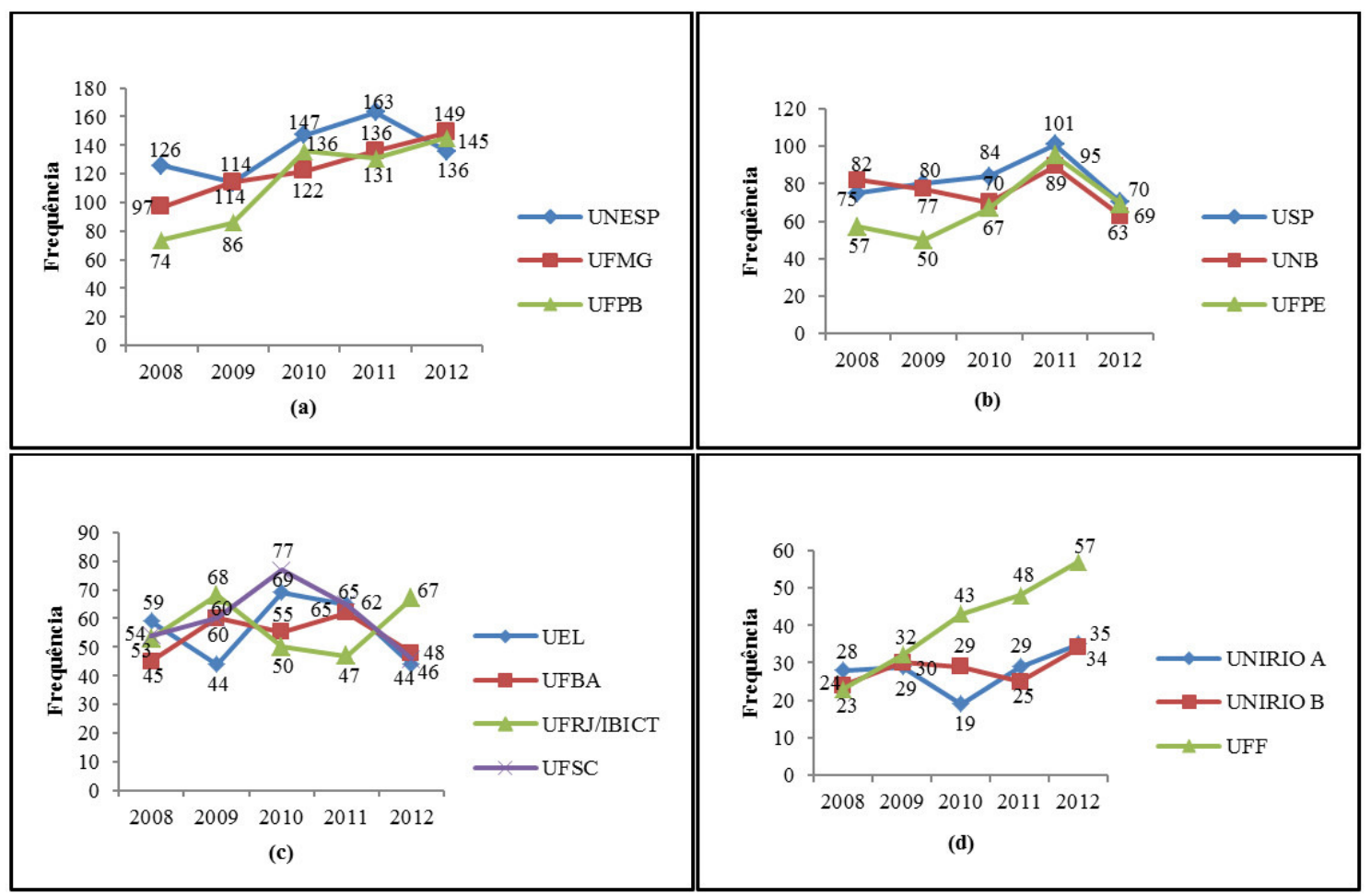

Fonte: AUTRAN (2015).

Até o momento foram demonstrados apenas os dados brutos de produção científica, contudo afirmam Andretta; Silva; Ramos (2012, p. 56): "Outra forma de ver a performance de uma instituição é por meio da produtividade," ou seja, a média resultante entre a quantidade de docentes e a quantidade de publicações.

Supunha-se, inicialmente, que a dimensão dos programas condicionava a produção científica dos mesmos. Entretanto, essa hipótese não é confirmada, pois à medida que o PPGCI da USP, com o quadro de 27 docentes, é comparado ao PPGCI UFMG com 28, demonstra produtividade inferior. Comparando-se também os PPGCIs que possuem entre 18 e 19 docentes, caso da UNESP, UFPB e UNB, constata-se que a produtividade do primeiro foi 36,1 , do segundo 30,1 e do terceiro 21,6 . Outra comparação pode ser feita entre os PPGCIs UNIRIO A, UFF e UFSC, enquanto o primeiro, com 12 docentes tem a média de produtividade 11,6 , o segundo com o mesmo número de professores tem a média 16,83 e o terceiro, com 13 docentes, apresenta a média de 23,23. (Tabela 3). 
Tabela 3 - Produtividade docente/ano

\begin{tabular}{lccc}
\hline PPGCI & $\begin{array}{c}\text { No de } \\
\text { docentes }\end{array}$ & $\begin{array}{c}\text { No de } \\
\text { produções }\end{array}$ & $\begin{array}{l}\text { Média de } \\
\text { produtividade por } \\
\text { docente/ano }\end{array}$ \\
\hline \hline UEL & 14 & 281 & 20,07 \\
UFBA & 14 & 270 & 19,28 \\
UFF & 12 & 201 & 16,83 \\
UFMG & 28 & 617 & 22,03 \\
UFPB & 19 & 572 & 30,10 \\
UFPE & 15 & 338 & 22,53 \\
UFRJ/IBICT & 16 & 285 & 17,81 \\
UFSC & 13 & 302 & 23,23 \\
UNB & 18 & 381 & 21,16 \\
UNESP & 19 & 686 & 36,10 \\
UNIRIO A & 12 & 140 & 11,60 \\
UNIRIO B & 10 & 142 & 14,20 \\
USP & 27 & 410 & 15,18 \\
\hline
\end{tabular}

Fonte: AUTRAN (2015).

\section{Considerações finais}

Presume-se que as informações aqui apresentadas, apenas de caráter quantitativo, possam- servir de insumo para melhor caracterização do perfil de produção acadêmica e de produtividade de cada programa, identificar deficiências e apontar características importantes da atuação docente com a área de Ciência da Informação.

A utilização da ferramenta sciptLattes se mostrou robusta e possibilitou apontar indicadores de produção e produtividade.

Quanto ao suporte documental mais utilizado para comunicação da ciência, os resultados coincidem com aqueles apresentados por Santilone et al.(2012). Em termos comparativos as autoras citadas referem que $41 \%$ dos docentes utilizaram as comunicações em eventos e $34 \%$ os artigos publicados em periódicos. Nesta investigação os resultados apontam 36,8\% para comunicações apresentadas em eventos e 34,3\% para artigos, sugerindo que, a exemplo das áreas Engenharia e Computação, os docentes demonstram a tendência de comunicar a ciência através deste suporte em detrimento da prática tradicional de comunicá-la através de periódicos.

Foi observado, ainda, que a dimensão do corpo docente não constitui condicionante para a produção e produtividade, considerando-se que PPGCIs com número idêntico de docentes apresentam desempenho diferenciado.

Como trabalhos futuros, pretende-se investigar a atuação dos PPGCIs por meio das redes de coautoria acadêmica, formação de recursos humanos, e a qualificação dos periódicos segundo o estrato Qualis. 


\section{Referências}

ALVES, A. D.; YANASSE, H. H.; SOMA, N. Y. SUCUPIRA: um sistema de extração de informações da Plataforma Lattes para edentificação de redes sociais acadêmicas In: CONFERÊNCIA IBÉRICA DE SISTEMAS E TECNOLOGIAS DE INFORMAÇÃO (CISTI) 6., 2011, Chaves. Anais...Chaves, Portugal: 2011a. Disponível em: <http://mtcm19.sid.inpe.br/col/sid.inpe.br/mtc-

m19/2011/12.14.17.28/doc/05974195.pdf>. Acesso em: 30 maio 20133.

ALVES, A. D.; YANASSE, H. H.; SOMA, N. Y. Perfil dos bolsistas PQ das áreas de engenharia de produção e de transportes do CNPq: enfoque na subárea de pesquisa operacional. In: SIMPÓSIO BRASILEIRO DE PESQUISA OPERACIONAL, 43., 2011, Ubatuba. Anais...Ubatuba: SOBRAPO, 2011b.

ALVES, A. D.; YANASSE, H. H.; SOMA, N. Y. LattesMiner: uma linguagem de domínio específico para extração automática de informações da Plataforma Lattes. In: WORKSHOP DE COMPUTÇÃO APLICADA (WORCAP) 12., 2011, São José dos Campos. Anais... São José dos ampos: INPE, 2011c. Disponível em: <http://mtc-m18.sid.inpe.br/col/sid.inpe.br/mtcm18/2013/01.15.16.10/doc/worcap2012_submission_61 - Alexandre D. Alves.pdf>. Acesso em: 8 jul. 2013

ALVES, V. B. A influência do open access nas comunidades acadêmicas da área de Biblioteconomia no nordeste do Brasil. 2011. 283f. Tese (Doutorado em Informação e Comunicação em Plataformas Digitais) Faculdade de Letras. Porto: Universidade do Porto, 2011.

ANDRETTA, P. I. S.; SILVA, E. G.; RAMOS, R. C. Aproximações sobre produção, produtividade e colaboração científica entre os departamentos de Ciência da Informação do Estado de São Paulo. Revista Digital de Biblioteconomia e Ciência da Informação, v. 9, n. 2, p. 49-63, 2012.

AUTRAN, M. M. M. Comunicação da ciência, produção científica e rede de colaboração acadêmica: análise dos Programas brasileiros de PósGraduação em Ciência da Informação. 2015. 407f. Tese (Doutorado em Informação e Comunicação em Plataformas Digitais) - Faculdade de Letras, Universidade do Porto, Porto, 2015.

BARATA, R. B.; GOLDBAUM, M. Perfil dos pesquisadores com bolsa de produtividade em pesquisa do CNPq da área de saúde coletiva. Cadernos de Saúde Pública, v. 19, n. 6, p. 1863-1876, 2003.

BASTOS, B. B.; SILVA, L. A comunidade científica no contexto da mediação comunicacional global: afecção das dinâmicas cognitivas e sociais. In: CONFERÊNCIA LUSÓFONA, 6., SOPCOM IBÉRICO, 4., 2009, Lisboa. Anais...Lisboa: Universidade Lusófona, 2009. Disponível em: <http://conferencias.ulusofona.pt/index.php/sopcom_iberico/sopcom_iber ico09/paper/view/307>. Acesso em: 15 abr. 2013 
BECHER, T.; TROWLER, P. Academic tribes and territories: intellectual enquiry and the cultures of disciplines. 2. ed. Buckingham: Open University Press, 2001.

BOMFÁ, C. R. Z.; SILVA, L. A Comunidade científica portuguesa: alguns dos recursos utilizados para a obtenção da visibilidade científica. In: CONGRESSO LUSOCOM - Conferência Lusófona, 8., 2009, Lisboa. Anais... Lisboa: Universidade Lusófona, 2009. Disponível em: <http://conferencias.ulusofona.pt/index.php/lusocom/8lusocom09/paper/ viewFile/198/174>. Acesso em: 15 abr. 2013.

BORGES, M. M. A esfera: comunicação acadêmica e novos media. 2006. 801f. Tese (Doutorado em Letras) - Faculdade de Letras, Universidade de Coimbra, Coimbra, 2006.

CAVALCANTE, R. A. et al. Perfil dos pesquisadores da área de Odontologia no Conselho Nacional de Desenvolvimento Científico e Tecnológico (CNPq). Revista Brasileira de Epidemiologia, v. 11, n. 1, p. 106-113, 2008.

COSTA, J. L. O. Padrões de comunicação em diferentes comunidades científicas. 2009. 187f. Tese (Mestrado em Ciência da Informação) Escola de Engenharia, Universidade do Minho, Guimarães, 2009.

CRONIN, B. Scholarly communication and epistemic cultures. New Review of Academic Librarianship, v. 9, n. 1, p. 1-24, 2003.

DANUELLO, J. C.; OLIVEIRA, E. F. T. Análise cientométrica: produção científica e redes colaborativas a partir das publicações dos docentes dos programas de graduação em Fonoaudiologia no Brasil. Em Questão, v. 18, n. especial, p. 65-79, 2012.

DAURA, A. P.; GALVÃO, M. C. B. O perfil docente e a interdisciplinaridade: estudando os cursos de graduação do campo da Ciência da Informação no Brasil. Revista EDICIC, v. 1, n. 3, p. 229-247, 2011.

DIGIAMPIETRI, L. A.; SILVA, E. E. A framework for social network of researchers analysis. Iberoamerican Journal of Applied Computing, v. $1, \mathrm{n}$. 1 , p. 1-24, 2011.

DOMINGOS, N. A. M. Análise da estrutura dos resumos de dissertações e teses em psicologia. In: WITTER, G. P. (Ed.). Produção científica em psicologia e educação. Campinas: Alínea, 1999. p. 181.

DUARTE, E. N.; SILVA, E. P.; ZAGO, C. C. Gestão do conhecimento: revelações sobre a produção científica. Informação \& Sociedade: estudos, v. 14, n. 2, p. 173-200, 2004.

FAYYAD, U.; PIATETSKY-SHAPIRO, G.; SMYTH, P. Advances in knowledge discovery and data mining. Cambridge: MIT Press, 1996.

FUNDAÇÃO DE AMPARO À PESQUISA DO ESTADO DE SÃO PAULO (FAPESP). Análise da produção científica a partir de indicadores 
bibliométricos. In: INDICADORES de ciência, tecnologia e inovação em São Paulo. São Paulo: FAPESP, 2010. v. 1. p. 4-8.

KNORR-CETINA, K. Epistemic cultures. Cambridge, MA: Harvard College, 1999.

KUHN, T. S. A estrutura das revoluções científicas. São Paulo: Perspectiva, 1978.

LANE, J. Let's make science metrics more scientific. Nature, v. 464, p. 488-489, 2010.

LE COADIC, Y. F. A Ciência da Informação. 2. ed. Brasília: Briquet de Lemos/Livros, 2004.

MARTELLI-JUNIOR, $\mathrm{H}$. et al. Pesquisadores do CNPq na área de Medicina: comparação das áreas de atuação. Revista da Associação Médica Brasileira, v. 56, n. 4, p. 478-483, 2010.

MEADOWS, A. J. A comunicação científica. Brasília: Briquet de Lemos/Livros, 1999.

MENA-CHALCO, J. P.; CESAR-JUNIOR, R. M. ScriptLattes: an open-source knowledge extraction system from the Lattes Platform. Journal of the Brazilian Computer Society, v. 15, n. 4, p. 31-39, dez. 2009.

MENA-CHALCO, J. P.; CESAR-JUNIOR, R. M. Prospecção de dados acadêmicos de currículos Lattes através de scriptLattes. In: HAYASHI, $M$. C. P. I.; LETA, J. (Orgs.). Bibliometria e Cientometria: reflexões teóricas e interfaces. São Carlos: Pedro \& João, 2011. p. 20.

MENA-CHALCO, J. P.; DIGIAMPIETRI, L. A.; CESAR-JUNIOR, R. M. Caracterizando as redes de coautoria de currículos Lattes. In: BRAZILIAN WORSHOP ON SOCIAL NETWORK ANALYSIS AND MINING (BRASNAM), $1 .$, 2012, Curitiba. Anais...Curitiba: Brazilian Computing Society, 2012. Disponível em: <http://professor.ufabc.edu.br/ jesus.mena/publications/pdf/menachalco 2012coautoria-lattes.pdf>. Acesso em: 5 jun. 2013.

MENA-CHALCO, J. P.; DIGIAMPIETRI, L. A.; OLIVEIRA, L. B. Perfil de produção acadêmica dos programas brasileiros de pós-graduação em Ciência da Computação nos triênios 2004-2006 e 2007-2009. Em Questão, v. 18, n. especial, p. 215-229, 2012.

MERTON, R. K. The sociology of science: theoretical and empirical investigations. Chicago: The University of Chicago, 1973.

MUELLER, S. P. M. A publicação da ciência: áreas científicas e seus canais preferenciais. DataGramaZero-Revista de Ciência da Informação, v. 6, n. $1,2005$.

MUELLER, S. P. M.; CAMPELLO, B. S.; DIAS, E. J. W. Disseminação da pesquisa em Ciência da Informação e Biblioteconomia no Brasil. Ciência da Informação, v. 25, n. 3, 1996. 
NORONHA, D. P.; POBLACIÓN, D. A. Producción académica de docentes/doctores de los programas de posgrado em Ciencia de la Información en Brasil. Ciências de la Información, v. 33, n. 1, p. 25-34, 2002.

OLIVEIRA, D. A influência da Ciência da Informação nos cursos de graduação em Biblioteconomia no Brasil: formação docente, aspectos teóricos e manifestações temáticas. 2011.185f. Tese (Doutorado em Ciência da Informação) - Escola de Ciência da Informação, Universidade Federal de Minas Gerais, Belo Horizonte, 2011.

PACHECO, R. C. S.; FORCELLINI, F.; KERN, V. M. Uma análise da pesquisa em Engenharia e Ciências Mecânicas no Brasil a partir dos dados da Plataforma Lattes. Revista ABCM Engenharia, v. 12, n. 1, p. 18-24, 2007.

PACHECO, R. C. S.; KERN, V. M.; STEIL, A. V. Aplicações de arquitetura conceitual em plataformas e-Gov: da gestão da informação pública à construção da sociedade do conhecimento. PontodeAcesso, v. 1, n. 1, p. 71-87, 2007.

PINHEIRO, L. V. R. Comunidades científicas e infra-estrutura tecnológica no Brasil para uso de recursos eletrônicos de comunicação e informação na pesquisa. Ciência da Informação, v. 32, n. 3, p. 62-73, 2003.

POLANIY, M. Science, faith and society. London: Oxford University Press, 1946.

POLANIY, M. The republic of science its political and economic theory. In: SHILS, E. (Ed.). Criteria for scientific development. Cambridge: MIT Press, 1968. p. 1-21.

SANTANA, G. A. et al. Indicadores científicos: uma análise da produção do programa de pós-graduação em Sociologia ( PPGS ) da UFPE a partir dos currículos da Plataforma Lattes (PL). In: ENCONTRO REGIONAL DE ESTUDANTES DE BIBLIOTECONOMIA, DOCUMENTAÇÃO, CIÊNCIA DA INFORMAÇÃO E GESTÃO DA INFORMAÇÃO, 14., 2011, São Luis. Anais... São Luis: UFMA, 2011

SANTILONE, M. A. et al. Mapeamento da produção científica dos docentes vinculados aos programas de pós-graduação em Ciência da Informação credenciados pela CAPES. CRB-8 Digital, v. 1, n. 5, p. 86-101, 2012.

SANTOS, R. N. M.; CÂNDIDO, L.; KUPPENS, C. Produtividade em pesquisa do CNPq: análise do perfil dos pesquisadores da Química. Química Nova, v. 33, n. 2, p. 489-495, 2010.

SANTOS, S. et al. Perfil dos pesquisadores da Saúde Coletiva no Conselho Nacional de Desenvolvimento Científico e Tecnológico. Physis, v. 19, n. 3, p. 761-775, 2009.

SCARPELLI, A. C. et al. Academic trajectories of dental researchers receiving CNPq's productivity grants. Brazilian Dental Journal, v. 19, n. 3, p. 252-256, 2008. 
SCHWARTZMAN, S. Um espaço para a ciência: a formação da comunidade científica no Brasil. Brasília: Ministério de Ciência e Tecnologia, 2001.

SILVA, A. O. et al. Colaboração entre programas de pós-graduação brasileiros em Ciência da Informação: modelagem baseada em grafos. Informação \& Informação, v. 17, n. 3, 2012a.

SILVA, F. M. et al. Proposta metodológica para a geração de indicadores científicos: análise dos programas de pós-graduação em ciência da informação (PPGCI) da região nordeste. Revista Digital de Bibloteconomia e Ciência da Informação, v. 10, n. 1, p. 67-90, 2012b.

SILVA, L. Implicações cognitivas e sociais da globalização das redes e serviços telemáticos: estudo das implicações da comunicação reticular na dinâmica cognitiva e social da comunidade científica. 2002. 693f. Tese (Doutorado em Ciências e Tecnologia da Comunicação) - Departamento de Comunicação e Arte, Universidade de Aveiro, Aveiro, 2002.

SZCZEPANIK, G. E. A concepção de comunidade científica segundo Thomas Kuhn. 2013. Disponível em: <http://www.paradigmas.com.br/parad27/p27.9.htm>. Acesso em: 1 maio 2013.

TARGINO, M. G.; GARCIA, J. C. R. Ciência brasileira na base de dados do Institute for Scientific Information (ISI). Ciência da Informação, v. 29, n. 1 , p. 103-117, 2000.

ZIMAN, J. M. Conhecimento público. São Paulo: EDUSP, 1979. 